Annuaire suisse de politique de développement

$17 \mid 1998$

Propriété intellectuelle : quels enjeux pour les pays en développement ?

\title{
La propriété intellectuelle et ses conséquences pour les pays en développement
}

\section{Carliene Brenner}

\section{(2) OpenEdition}

\section{Journals}

Édition électronique

URL : http://journals.openedition.org/aspd/721

DOI : $10.4000 /$ aspd. 721

ISSN : 1663-9669

Éditeur

Institut de hautes études internationales et du développement

Édition imprimée

Date de publication : 1 avril 1998

Pagination : 7-24

ISSN : 1660-5934

Référence électronique

Carliene Brenner, "La propriété intellectuelle et ses conséquences pour les pays en développement », Annuaire suisse de politique de développement [En ligne], 17 | 1998, mis en ligne le 21 juillet 2012,

consulté le 07 septembre 2020. URL : http://journals.openedition.org/aspd/721 ; DOI : https://doi.org/ $10.4000 / a s p d .721$ 


\title{
LA PROPRIÉTÉ INTELLECTUELLE ET SES CONSÉOUENCES POUR LES PAYS EN DÉVELOPPEMENT
}

CARLIENE BRENNER*

\begin{abstract}
E PRÉSENT ARTICLE évalue les conséquences que pourrait entraîner pour les pays en développement une protection renforcée de la propriété intellectuelle telle qu'elle est prévue dans l'Accord sur les aspects des droits de propriété intellectuelle liés au commerce (ADPIC). Le premier chapitre dresse un bilan du renforcement et de l'extension de cette protection au cours de ces dernières années. Les deux chapitres suivants présentent les différents types et les différentes portées des droits de propriété intellectuelle et le rôle des principales institutions actives dans ce domaine. Le quatrième chapitre résume les principales dispositions et exceptions de l'ADPIC. Le cinquième chapitre part du cas de la biotechnologie appliquée dans l'agriculture pour étudier les effets potentiels d'une extension des droits de propriété intellectuelle à des domaines jusqu'alors non protégés: micro-organismes, variétés animales et végétales. Le dernier chapitre explique l'impossibilité de tirer des conclusions définitives: il avance que l'impact global ne sera ni entièrement positif ni entièrement négatif et souligne le rôle que doit jouer la politique gouvernementale dans l'atténuation des éventuels effets négatifs.
\end{abstract}

\section{DROITS DE PROPRIÉTÉ INTELLECTUELLE: DE L'ANTIQUITÉ À L'ADPIC}

Les droits de propriété intellectuelle peuvent être définis comme un ensemble de lois spécialement prévues pour protéger ou récompenser les inventeurs ou les créateurs d'un nouveau savoir. Contrairement aux biens matériels, le savoir peut être partagé par un nombre indéfini de personnes sans jamais perdre de sa valeur. Les inventeurs dépendent donc entièrement de la protection juridique pour éviter que leur invention soit tout simplement copiée et utilisée sans compensation pour eux. Les droits de propriété intellectuelle visent ainsi à conférer des droits exclusifs aux inventeurs pour une durée limitée (Lesser, 1994).

L'idée de protéger la propriété intellectuelle remonte à l'Antiquité. Dans la Grèce antique, les commerçants et les inventeurs jouissaient de droits de monopole déjà deux cents ans avant Jésus-Christ. Les inventeurs bénéficiaient aussi de privilèges très similaires à ceux d'un brevet dans l'Europe du Moyen-Age entre le $\mathrm{XI}^{\mathrm{e}}$ et le XII ${ }^{e}$ siècle, soit à une période où la production et la commercialisation étaient organisées autour des artisans et du système de guildes (Juma, 1989). Depuis la Révolution industrielle, différentes formes de protection des brevets sont appliquées en Europe. L'Allemagne s'est par exemple dotée d'une loi moderne sur le droit des brevets en 1877 . 
Les lois sur les brevets sont d'essence nationale et peuvent donc varier grandement d'un pays à l'autre. Le premier effort visant à harmoniser les droits de propriété intellectuelle au niveau international a débouché sur la signature, à Paris en 1883, de la Convention internationale sur la protection de la propriété industrielle. La Convention de Paris est devenue un symbole sur la voie de l'internationalisation des droits de propriété intellectuelle car elle contient le principe de la «non-discrimination». Selon ce principe, tout Etat contractant doit accorder aux ressortissants d'autres pays la même protection qu'à ses propres citoyens. Depuis 1883, divers accords internationaux portant sur les droits de propriété intellectuelle sont entrés en vigueur. Voici les plus importants d'entre eux: le Traité de Budapest, la Convention sur le brevet européen et le Traité de coopération en matière de brevets ${ }^{1}$.

En 1988, 115 pays ont accepté que les inventions industrielles bénéficient d'une protection de la propriété intellectuelle, quelle que soit sa forme. Il convient toutefois de relever que près de la moitié de ces pays excluent de cette protection les inventions biologiques, c'est-à-dire les variétés animales et végétales (Lesser, 1991).

La protection des droits intellectuels appliquée aux ressources phytogénétiques et aux variétés végétales n'a pas suivi la même évolution, notamment en raison de la complexité et de la difficulté qu'implique la protection de la matière vivante. Un premier grand pas dans ce sens a été franchi aux Etats-Unis dans les années 30 avec l'introduction des variétés de maïs hybrides. Ces nouvelles variétés assuraient des rendements beaucoup plus élevés que les variétés à pollinisation libre, mais ne pouvaient pas servir de semence la saison suivante sans que l'on assiste à une baisse considérable du rendement. Ainsi, les agriculteurs qui avaient pour coutume de conserver une partie de leur récolte comme semence pour la saison suivante ont été obligés d'acheter chaque année des semences de ces nouvelles variétés pour maintenir leurs rendements. Ce système assurait une protection équivalant à un brevet aux inventeurs ou aux sélectionneurs des variétés hybrides.

Avec les progrès de la recherche agricole et de la sélection moderne des végétaux, les sélectionneurs en sont peu à peu venus à réclamer des droits de propriété intellectuelle pour protéger le fruit de leurs efforts. Ils appuyaient cette demande en arguant que leur contribution à la société devait être reconnue au même titre que celle des inventeurs industriels.

Fondés sur la Convention de Paris, les efforts des sélectionneurs ont été récompensés: certains pays ont adopté dans les années 20 - les Etats-Unis dans les années 30 - une législation visant à protéger les nouvelles variétés végétales. Aux Etats-Unis, le «Plant Patent Act» (loi sur les brevets sur les végétaux) de 1930 a admis la protection des végétaux obtenus par reproduction non sexuée (à

1. Le Traité de 1997 sur la reconnaissance internationale du dépôt des micro-organismes aux fins de la procédure en matière de brevets régit la reconnaissance du dépôt d'organismes vivants dans le cadre du système de protection ratifié au niveau international. La Convention sur le brevet européen s'applique aux membres de l'Union européenne. Le Traité de coopération en matière de brevets a pour but de simplifier et de rendre moins coûteuse l'obtention de brevets dans un grand nombre de pays par le dépôt d'une seule demande internationale auprès d'un des Etats membres. La procédure comprend ensuite la publication de la demande et un rapport d'enquête international (destiné à vérifier le caractère unique de la demande). Ces divers accords internationaux sur les brevets sont administrés par l'Organisation mondiale de la propriété intellectuelle (OMPI). 
l'exception des tubercules). Les végétaux issus d'une reproduction sexuée étaient exclus de cette protection puisque l'on considérait qu'ils ont la particularité d'évoluer et de se modifier au fil des générations et qu'il devient difficile de savoir sur quel élément portait le brevet à l'origine.

Les premiers efforts consentis au niveau international pour étendre et harmoniser les droits d'obtention - que l'on nomme aussi protection des variétés végétales - ont été entrepris en 1956 à l'occasion du congrès de l'Association internationale des sélectionneurs pour la protection des obtentions végétales (ASSINSEL). Cette initiative a conduit à l'adoption, en 1961, de la première convention internationale dans ce domaine, plus connue sous le nom d'Union pour la protection des obtentions végétales (UPOV).

Tandis que la forme des droits de propriété intellectuelle dans l'industrie et dans l'agriculture suivaient des voies différentes, on a assisté, au cours des années, à un renforcement progressif mais significatif de la protection intellectuelle dans tous les domaines de l'innovation. Cette évolution résulte notamment du mécontentement des titulaires de brevets et des pertes qu'ils ont essuyées du fait de la violation des droits de protection intellectuelle. La Commission américaine sur le commerce international a en effet estimé que les violations de brevets, de droits d'auteur et de marques appartenant à des inventeurs des pays industrialisés ont coûté quelque 61 milliards de dollars aux compagnies américaines en 1990 (Lesser, 1991).

Outre le fait que la protection de la propriété intellectuelle se renforce, l'avènement des biotechnologies a provoqué un rapprochement entre les formes de protection des innovations industrielles et biologiques, du moins dans les pays membres de l'OCDE. Le début de cette tendance a été marqué par la décision Chakrabarty prise par la Cour suprême des Etats-Unis en 1980, qui a autorisé pour la première fois l'octroi d'un brevet sur un organisme génétiquement modifié - ou forme de vie. Le mouvement était dès lors lancé: la première demande de brevet pour un végétal transgénique a été déposée en 1983, le premier brevet industriel portant sur une variété végétale a été accordé en 1985 aux Etats-Unis et en 1988 en Europe. Dans les années 80, le nombre de demandes de brevet dans le domaine des biotechnologies végétales a augmenté pour atteindre environ 250 par an (Joly, de Looze, 1996). (Le chapitre 2, consacré au type et à l'étendue des droits de propriété intellectuelle, montre que la révision de 1991 de la Convention de 1'UPOV a également contribué à aligner ces droits sur les droits des brevets.)

Les Etats-Unis ayant lourdement insisté sur le fait que l'absence d'une législation exhaustive sur les brevets et d'autres lois sur la propriété intellectuelle constitue une barrière non tarifaire au commerce, la communauté internationale a accepté, malgré l'opposition des pays en développement, d'inclure «la propriété intellectuelle qui touche au commerce» dans le Cycle d'Uruguay des négociations sur le commerce multilatéral, négociations lancées par l'Accord général sur les tarifs douaniers et le commerce (GATT). Ces efforts pour renforcer et étendre les droits de propriété intellectuelle ont débouché sur la signature de l'ADPIC (Accord sur les aspects de la propriété intellectuelle qui touchent au commerce; annexe $1 \mathrm{C}$ de l'Accord de Marrakech du $1^{\mathrm{er}}$ janvier 1995 instituant l'Organisation mondiale du commerce, OMC). La conclusion de cet accord signifie que le débat et les négociations sur les droits de la propriété intellec- 
tuelle ont changé de cadre: ils ne se déroulent désormais plus dans l'esprit technique de l'Organisation mondiale pour la propriété intellectuelle (OMPI), qui est une institution spécialisée des Nations Unies, mais dans le cadre de l'OMC récemment créée.

Les conséquences probables d'un renforcement des droits de propriété intellectuelle et de l'extension de la protection à des domaines non couverts jusqu'alors - notamment dans les pays en développement - tels que les micro-organismes, le matériel phytogénétique et les techniques utilisées pour les manipulations génétiques ont suscité un vif débat. Ce débat illustre bien les préoccupations que soulèvent la dégradation de l'environnement et la nécessité de protéger la diversité biologique et, plus formellement, l'application de l'Engagement international sur les ressources phytogénétiques de la FAO et de la Convention sur la diversité biologique. C'est ainsi que les enjeux du débat sur les droits de propriété intellectuelle et sur le matériel phytogénétique sont désormais liés aux «droits des agriculteurs» dans le cas de l'Engagement de la FAO et au «partage équitable des avantages obtenus à partir des ressources phytogénétiques » dans le cas de la Convention sur la diversité biologique.

$\square$ ASTM 98, l'article de P. Egger et de J. Benz.

\section{LES PRINCIPALES FORMES DES DROITS DE PROPRIÉTÉ INTELLECTUELLE ET LEUR ÉTENDUE}

Parmi les principales formes des droits de propriété intellectuelle, nous retenons ici les brevets, les droits d'obtention et les secrets commerciaux et n'entrons volontairement pas en matière sur les autres formes de protection que sont les droits d'auteur et les marques.

\subsection{BREVETS}

Les brevets, y compris les modèles déposés ou modèles d'utilité, constituent la plus courante et aussi la plus ancienne forme de protection de la propriété intellectuelle. Toute invention qui n'est pas expressément interdite peut être brevetée à condition de remplir les exigences décrites ci-après. Les découvertes, les théories scientifiques et les formules mathématiques sont exclues du domaine du brevetable, de même que les éléments réputés contraires à la morale. De plus en plus de pays appliquent des brevets à une gamme croissante d'inventions (produits et procédés) qui comprennent des matières vivantes sélectionnées telles que des séquences d'ADN, des gènes, des micro-organismes, des parties de végétaux ainsi que des variétés animales et végétales. Nombre de pays en développement et certains Etats membres de l'OCDE ont toutefois exclu les produits pharmaceutiques et les produits agricoles du domaine des brevets.

Pour prétendre à un brevet, l'invention considérée doit remplir les trois grandes conditions suivantes: elle doit être susceptible d'application industrielle; elle doit être nouvelle en ce sens qu'elle ne doit pas avoir été déjà publiée; elle doit être non évidente, ou impliquer une activité inventive, de sorte que l'invention apporte un savoir nouveau basé sur les connaissances antérieures. 
La condition de l'utilité (application industrielle) implique qu'il n'est pas possible de faire breveter de simples idées ou conceptions et que l'invention doit comprendre un effort humain qui pourrait - s'il était mis en œuvre - fonctionner comme prévu. La caractéristique de la nouveauté signifie qu'avant le dépôt de la demande l'invention n'était pas connue et qu'elle représente donc une découverte pour l'humanité.

La publication constitue par ailleurs une condition supplémentaire à l'octroi d'un brevet. Elle signifie que la demande de brevet doit contenir une description suffisamment claire et détaillée de l'invention pour permettre à une personne «du métier» de reproduire et d'utiliser le brevet ou de recréer l'invention (Lesser, 1991). Cependant, dans le cas d'un nombre croissant d'inventions relevant de la microbiologie, la description ne suffit pas à elle seule. On requiert alors le dépôt d'un échantillon du matériel biologique dont l'invention a été dérivée.

Ce sont en général les offices nationaux des brevets qui sont compétents pour établir si les demandes de brevets remplissent les exigences requises et pour accepter ou rejeter les demandes. La décision d'accorder un brevet (ou une autre forme de protection) se fonde sur une procédure d'examen ou d'enregistrement. Dans le cas d'un examen, on procède à une recherche dans les registres nationaux des brevets pour vérifier la nouveauté de l'invention faisant l'objet de la demande. La demande fait également l'objet d'un examen visant à établir si l'invention est suffisamment différente (ou non évidente) de l'état actuel des connaissances dans le domaine et constitue donc véritablement une invention. Dans le cas d'un enregistrement, une demande est reçue et enregistrée mais rien n'est entrepris pour apprécier la validité du brevet.

L'octroi d'un brevet confère à son titulaire, ou inventeur, un droit de monopole sur l'utilisation et sur les bénéfices de son invention pendant une période déterminée. Bien que la durée de protection varie d'un pays à l'autre, le brevet est protégé pendant une période qui se situe en général entre quatorze et vingt ans. (La durée de protection d'un modèle déposé est plus brève: le plus souvent entre quatre et sept ans.) Pendant cette période, l'inventeur (titulaire du brevet) a un droit d'exclusivité sur l'exploitation de son invention (fabrication, utilisation, vente ou importation). Il peut aussi exiger le versement d'une rémunération en échange du droit d'exploitation de l'invention (licence; Lesser, 1991).

Dans certains cas, une licence peut être concédée sur une invention brevetée sans l'accord du titulaire du brevet («licence obligatoire»). Une telle licence peut être accordée en raison d'une «exploitation insuffisante» du brevet ou lorsque l'on considère qu'il y va de l'intérêt public, notamment lorsqu'il s'agit de la santé, de l'alimentation ou de la sécurité (Lesser, 1991).

\subsection{DROITS D'OBTENTION}

Tandis que la plupart des pays - à l'exception notable des Etats-Unis - excluent les inventions «végétales» de la protection conférée par les brevets d'utilité, un nombre croissant d'entre eux autorisent une solution de rechange pour les protéger: les droits d'obtention. Conformément à la Convention de l'UPOV - entrée en vigueur en 1961 et révisée en 1972, en 1978 et en 1991 les droits d'obtention se limitent aux nouvelles variétés végétales².

2. «On entend par «variété» un ensemble végétal d'un taxon botanique du rang le plus bas connu qui, qu'il réponde 
Les droits d'obtention offrent une protection contre l'utilisation non autorisée de variétés protégées. Les exigences à remplir pour bénéficier de la protection d'une variété végétale sont les mêmes que pour les brevets d'utilité, tout en étant moins étendues. Elles comprennent cependant les caractéristiques suivantes: nouveauté, distinction, uniformité ou homogénéité et stabilité. Une autre exigence veut aussi que la variété porte une dénomination permettant de l'identifier.

La variété pour laquelle une protection est demandée est réputée nouvelle si elle n'a pas été mise en vente ou commercialisée dans le pays où la demande est déposée, ou dans un autre pays, pendant plus de quatre ans. Pour remplir le critère de la distinction, qui constitue le fondement des droits d'obtention, la variété doit se distinguer clairement, par une ou plusieurs caractéristiques importantes, de toute autre variété notoirement connue. La variété est réputée homogène si ses caractères pertinents demeurent suffisamment uniformes dans une même plantation et stable si ses caractères pertinents restent inchangés à la suite de ses reproductions ou multiplications successives ${ }^{3}$.

Les droits d'obtention sont certes considérés comme une forme moins sûre de protection de la propriété intellectuelle que le brevet, mais chaque nouvelle révision de la Convention de l'UPOV a renforcé l'étendue de la protection accordée aux obtenteurs. La dernière révision, qui remonte à 1991, diffère beaucoup en bien des points de la version précédente en vigueur depuis 1978. Voici les principales différences : étendue et durée de la protection, droits d'obtention, privilège des paysans et apparition de la notion de «variété essentiellement dérivée».

A l'article 3, la Convention de 1991 exige des Etats contractants qu'ils accordent une protection pour tous les genres et espèces, tandis que la convention de 1978 limitait le nombre des espèces à protéger. Les pays déjà membres de la convention bénéficiaient d'un délai de cinq ans pour étendre la protection prévue; pour les nouveaux membres, ce délai a été prolongé à 10 ans. Pour ce qui est de la durée de la protection, elle est fixée à 25 ans pour les arbres et la vigne et à 20 ans pour tous les autres végétaux selon la convention de 1991. Cette durée était fixée à 18 et à 15 ans respectivement dans la convention de 1978.

Alors que les droits d'obtention ne couvraient que la production et la vente du matériel de reproduction ou de multiplication végétative de la variété protégée dans la convention de 1978, ils s'étendent selon la convention de 1991 au produit de la récolte (p. ex. au fruit d'une variété protégée d'arbres fruitiers) ou, en d'autres termes, à toute production et reproduction de la variété protégée. Les pays peuvent cependant, dans des limites raisonnables, restreindre les droits d'obtention afin d'assurer la pérennité de formes traditionnelles de conservation des semences à la ferme.

Les droits d'obtention s'appliquent certes à la production à des fins commerciales, mais ne s'étendent pas à la production du matériel de reproduction qui n'est pas destiné à la commercialisation. La production de semences par un

ou non pleinement aux conditions pour l'octroi d'un droit d'obtenteur, peut être défini par l'expression des caractères résultant d'un certain génotype ou d'une certaine combinaison de génotypes, distingué de tout autre ensemble végétal par l'expression d'au moins un desdits caractères et considéré comme une entité eu égard à son aptitude à être reproduit conforme. » Convention de l'UPOV, version 1991, article 1.

3. Voir les articles 6 à 9 de la Convention de l'UPOV. 
agriculteur aux fins d'un semis ultérieur sur sa propre exploitation ne tombe donc pas dans le domaine des droits d'obtention. Cette exception notable s'appelle le «privilège du paysan».

Tant la convention de 1978 que celle de 1991 prévoient une «exception aux droits d'obtention» qui autorise l'utilisation d'une variété protégée à titre expérimental ou aux fins de la création de nouvelles variétés, sans l'autorisation de l'obtenteur. La convention de 1991 introduit toutefois la notion de «dérivation essentielle» et prévoit que les variétés «essentiellement dérivées » d'une variété protégée peuvent être protégées mais ne peuvent pas être commercialisées sans l'autorisation de l'obtenteur de la variété protégée ${ }^{4}$.

Pour l'heure, c'est la convention de 1978 qui s'applique. En effet, la convention de 1991 n'entrera en vigueur qu'un mois après avoir été ratifiée par cinq Etats. Or, seuls trois pays membres l'ont ratifiée jusqu'ici. Les membres de l'UPOV avaient par ailleurs prévu qu'après l'entrée en vigueur de la convention de 1991, aucune adhésion ne serait plus admise à la convention de 1978. Cependant, puisqu'un certain nombre de pays avaient entamé des procédures d'adhésion à la convention de 1978, celle-ci restera ouverte à de nouveaux membres pendant une année encore après l'entrée en vigueur de la convention de 1991. Les trois seuls pays qui avaient adhéré à la convention de 1991 en juin 1997 étaient le Danemark, les Pays-Bas et Israël.

\subsection{SECRET COMMERCIAL}

Le secret commercial peut être défini comme une information qui assure un avantage concurrentiel à son détenteur et qui est tenue secrète plutôt que divulguée publiquement. Dans la plupart des pays, le secret commercial n'est pas défini dans la loi, bien que la législation autorise ceux dont les secrets industriels ont été acquis indûment à ester en justice pour mettre fin à l'utilisation de ces secrets par des tiers et/ou pour exiger un dédommagement. En principe, le secret commercial est protégé par une restriction de l'accès à l'information, cette restriction étant définie par des contrats ou d'autres accords. Les personnes concernées peuvent en faire usage pour garder le contrôle de certains aspects non codifiés de la technologie - technologie dite «implicite»-qui augmente la valeur du brevet. La vente ou le transfert de technologies complexes fait ainsi le plus souvent l'objet d'un contrat global comprenant une ou plusieurs licences, des secrets commerciaux et des conseils en matière de gestion (Lesser, 1991).

Les lignées généalogiques d'hybrides ou des réactifs spécifiques utilisés lors de la manipulation génétique peuvent être protégés par le secret commercial plutôt que par un brevet ou par une mesure de protection des variétés végétales. Lorsqu'une technologie ne bénéficie pas encore d'une autre forme de protection, les entreprises chercheront souvent à la protéger par le secret commercial.

4. «Avec le génie génétique, il est désormais possible de transformer n'importe quelle variété en laboratoire en lui ajoutant un ou plusieurs gènes. De plus, la nouvelle variété ainsi obtenue est protégée si elle se distingue clairement de la variété initiale, le dernier innovateur n'ayant aucune obligation envers l'obtenteur initial» (traduction), in: Why Change the UPOV Convention? The Evolution of the Convention Resulting from the 1991 Act, présentation par Adelaide Harries lors d'une séance d'information sur la protection de nouvelles variétés végétales selon la Convention de l'UPOV, Rome, 19 avril 1996. 


\subsection{ACCORDS DE TRANSFERT DE MATÉRIEL}

Les accords de transfert de matériel sont largement utilisés pour transférer du matériel génétique à des fins expérimentales. On en fait un usage courant dans le cadre de recherches menées conjointement, en particulier dans des projets de recherche financés par l'Etat ou par des donateurs et dans des programmes faisant intervenir des universités et/ou des instituts de recherche publics, mais aussi dans des travaux de recherche impliquant des partenaires du secteur privé.

\section{RÔLE DES PRINCIPALES INSTITUTIONS RÉGISSANT LES DROITS DE PROPRIÉTÉ INTELLECTUELLE}

L'ADPIC ne déroge nullement aux obligations qui découlent des dispositions de la Convention de Paris (pour la protection de la propriété intellectuelle), de la Convention de Berne (pour la protection des œuvres littéraires et artistiques), de la Convention de Rome (sur la protection des artistes interprètes ou exécutants, des producteurs de phonogrammes et des organismes de radiodiffusion) ou du Traité sur la propriété intellectuelle en matière de circuits intégrés. Jusqu'ici, les négociations touchant aux droits de propriété intellectuelle se sont principalement déroulées dans le cadre de l'OMPI, mais d'autres organisations importantes telles que l'Organisation mondiale du commerce (OMC) ou l'UPOV continueront de jouer un rôle important, voire croissant, dans ce domaine.

Créée en 1967, l'OMPI est devenue une institution spécialisée des Nations Unies en 1974. Elle a pour tâche de promouvoir la protection de la propriété intellectuelle à travers le monde par la coopération des Etats et, s'il y a lieu, en collaboration avec toute autre organisation internationale. Elle assure également la coopération administrative entre les unions de propriété intellectuelle, c'est-àdire les «unions» créées par les conventions de Paris et de Berne et par plusieurs traités annexes complémentaires conclus par les membres de l'Union de Paris.

L'OMPI offre tout un éventail de services aux pays en développement dans le domaine de la propriété intellectuelle. Ces services comprennent des conseils et des cours de formation proposés aux gouvernements et aux organisations des secteurs public et privé et portent sur les négociations et la conclusion d'accords concernant les licences sur la propriété intellectuelle et sur la gestion de cette propriété lorsque de tels accords ont un impact sur l'environnement. Ces services incluent aussi des rapports de recherche sur l'état des technologies dans divers domaines techniques. Ces rapports se fondent sur les informations publiées dans les documents du brevet et sont fournis gratuitement.

Le $1^{\text {er }}$ janvier 1996, l'OMPI et l'OMC ont conclu un accord selon lequel l'OMPI continuera de fournir une assistance technique et juridique aux pays en développement et maintiendra sa coopération technique avec eux. Elle étendra par ailleurs ses activités d'assistance dans le domaine de l'ADPIC aux pays en développement qui sont membres de l'OMC mais ne sont pas nécessairement membres de l'OMPI.

Comme par le passé, l'OMPI concentrera ses activités en faveur des pays en développement sur le droit de la propriété industrielle. La protection des variétés 
végétales reste en effet dans le giron de l'UPOV, qui sera appelée à jouer un rôle plus important puisque les pays à opter pour la solution des droits d'obtention sont plus nombreux que ceux qui choisissent la voie des brevets pour protéger les variétés végétales.

Cependant, tandis que l'OMC, et par conséquent l'ADPIC, réunit presque tous les Etats de la planète, l'UPOV ne comptait que 34 Etats membres en août 1997. Ceux-ci comprennent presque tous les pays de l'OCDE, à l'exception de la Grèce, de l'Islande, de la Corée, du Luxembourg et de la Turquie. Et voici les seuls pays membres de l'UPOV qui ne sont pas membres de l'OCDE: Afrique du Sud, Argentine, Chili, Colombie, Equateur, Israël, Paraguay, Slovaquie, Ukraine et Uruguay.

Un nombre croissant de pays d'Europe de l'Est, d'Amérique latine, du MoyenOrient, d'Afrique et d'Asie se préparent toutefois à adhérer à l'UPOV ou sont en train de réviser leurs lois sur la protection des semences et des végétaux. Certains pays choisissent d'adhérer à la Convention de l'UPOV de 1978, d'autres à celle de 1991. Dans l'ensemble, on prévoit donc une augmentation rapide des effectifs de l'UPOV, qui devrait compter environ 50 pays d'ici à 1999 et environ 80 en l'an 2000.

\section{DISPOSITIONS ET EXCEPTIONS DE L'ACCORD ADPIC}

L'Accord sur les aspects des droits de propriété intellectuelle qui touchent au commerce (ADPIC), entré en vigueur le $1^{\text {er }}$ janvier 1995, représente le premier effort universel pour obliger les pays à respecter certaines normes en matière de disponibilité, d'étendue et d'usage des droits de propriété intellectuelle. L'accord couvre tous les principaux domaines de la propriété intellectuelle, y compris le droit d'auteur et les droits connexes, les marques de fabrique ou de commerce, les indications géographiques, les dessins et modèles industriels, les brevets, les schémas de configuration (topographies) de circuits intégrés et la protection des renseignements non divulgués.

Selon les objectifs déclarés de l'ADPIC (article 7), la protection et le respect des droits de propriété intellectuelle devraient contribuer à la promotion de l'innovation technologique et au transfert et à la diffusion de la technologie, à l'avantage mutuel de ceux qui génèrent et de ceux qui utilisent des connaissances techniques et d'une manière propice au bien-être social et économique, et à assurer un équilibre de droits et d'obligations.

L'article 27 de l'accord prévoit qu'un brevet pourra être obtenu pour toute invention, de produit ou de procédé, dans tous les domaines technologiques, à condition qu'elle soit nouvelle, qu'elle implique une activité inventive et qu'elle soit susceptible d'application industrielle. L'article indique en outre que des brevets pourront être obtenus et qu'il sera possible de jouir de droits de brevet sans discrimination quant au lieu d'origine de l'invention, au domaine technologique et au fait que les produits sont importés ou sont d'origine nationale.

Une disposition prévoit cependant que les membres pourront exclure de la brevetabilité les inventions pouvant représenter un danger pour l' «ordre public» et les méthodes diagnostiques, thérapeutiques et chirurgicales pour les traitements des personnes et des animaux. Pourront également être exclus les végétaux et les 
animaux autres que les micro-organismes ainsi que les procédés essentiellement biologiques d'obtention de végétaux ou d'animaux autres que les procédés non biologiques et microbiologiques. Les membres doivent toutefois prévoir la protection des variétés végétales «par des brevets, par un système sui generis efficace ou par une combinaison de ces deux moyens».

L'article 28 définit les droits exclusifs conférés au titulaire du brevet. Ces droits empêchent des tiers de fabriquer, d'utiliser ou de vendre l'invention brevetée, qu'il s'agisse d'un produit ou d'un procédé, sans le consentement du titulaire du brevet. Celui-ci a également le droit de céder le brevet ou de le transmettre par voie successorale et de conclure des contrats de licence.

Les articles 65 et 66 contiennent des dispositions particulières pour les pays en développement membres de l'accord. Il s'agit notamment de dispositions transitoires et du délai dont disposent les pays pour se conformer à leurs engagements. En effet, les pays industrialisés bénéficient d'une année après l'expiration du délai d'entrée en vigueur pour appliquer les dispositions de l'Accord sur l'OMC ( $1^{\text {er }}$ janvier 1995), tandis que les pays en développement bénéficient pour ce faire d'une période additionnelle de quatre ans.

Quant aux pays les moins avancés, ils disposent d'une période encore plus longue (dix ans) pour appliquer les dispositions de l'ADPIC et le Conseil de l'ADPIC est même autorisé à prolonger encore ce délai.

L'ADPIC contient également une disposition autorisant l'octroi de licences obligatoires (comme indiqué dans le chapitre 2, il s'agit de licences accordées sans l'autorisation du titulaire du brevet). L'utilisation sans autorisation du titulaire du brevet pourra ainsi être permise à un candidat utilisateur lorsque ses efforts pour obtenir l'autorisation du détenteur du droit, suivant des conditions et modalités commerciales raisonnables, n'ont pas abouti dans un délai raisonnable. Cette disposition est toutefois soumise à un certain nombre de conditions visant à protéger les intérêts légitimes du titulaire du brevet. Dans des cas exceptionnels d'utilisation publique à des fins non commerciales ou dans des situations d'urgence nationale, il est toutefois possible de déroger à l'obligation de commencer par requérir l'obtention volontaire d'une licence.

\subsection{DISPOSITIONS PARTICULIÈRES DE L'ADPIC SUR LE TRANSFERT DE TECHNOLOGIE}

Outre l'objectif général mentionné ci-dessus de promouvoir l'innovation technologique ainsi que le transfert et la diffusion de technologie, l'ADPIC exige expressément des pays développés qu'ils offrent des incitations aux entreprises et institutions basées sur leur territoire afin de promouvoir et d'encourager le transfert de technologie vers les pays les moins avancés membres de l'accord (article 66).

Les exigences en matière de divulgation que doit satisfaire le déposant d'une demande de brevet (c'est-à-dire qu'il divulgue l'invention d'une manière suffisamment claire et complète pour qu'une personne du métier puisse l'exécuter; article 29) ont une série de conséquences cruciales pour l'accès à la technologie et à son transfert. Premièrement, pendant toute la durée de protection, il est possible de connaître la personne qui peut fournir la technologie. Deuxièmement, après expiration du brevet, l'invention divulguée tombe dans le domaine public 
et est librement disponible pour tous. Enfin, troisièmement, l'article 30, qui définit des «exceptions limitées» aux droits conférés, prévoit l'utilisation d'une invention à des fins expérimentales - c'est-à-dire dans le domaine de la recherche.

Par ailleurs, lorsqu'une technologie, qu'elle soit brevetée ou non, est aux mains d'un gouvernement, l'ADPIC permet à ce gouvernement de transférer cette technologie à des conditions préférentielles s'il le désire. De même, l'accord ne contient aucune disposition qui empêcherait un gouvernement ou une institution financière internationale d'appuyer financièrement le transfert volontaire et à des conditions préférentielles d'une technologie protégée appartenant à des privés.

\subsection{LE CHOIX DES PAYS EN DÉVELOPPEMENT EN MATIËRE DE PROTECTION DE LA PROPRIÉTÉ INTELLECTUELLE}

La plupart des pays en développement accordent des brevets pour protéger la propriété industrielle, mais peu d'entre eux ont jusqu'ici prévu une protection de la propriété intellectuelle pour du matériel phytogénétique et/ou pour des variétés végétales. L'engagement pris par ces pays aux termes de l'ADPIC offre toutefois un certain nombre de possibilités. En voici trois:

- autoriser la brevetabilité de tout matériel phytogénétique, y compris les variétés végétales;

- prévoir deux formes de protection, comme c'est le cas aux Etats-Unis: brevet pour le matériel phytogénétique et droits d'obtention ou un système sui generis pour la protection des variétés végétales;

- exclure le matériel phytogénétique de la brevetabilité et élaborer un système sui generis, ou adhérer à l'UPOV.

\section{DROITS DE PROPRIÉTÉ INTELLECTUELLE, BIOTECHNOLOGIES ET AGRICULTURE}

Dans le présent chapitre, nous considérons le cas des biotechnologies dans l'agriculture pour répondre à la question suivante: le renforcement et l'extension des droits de propriété intellectuelle vont-ils faciliter ou entraver le transfert de technologie et l'innovation dans le secteur agricole des pays en développement?

Pour commencer, il importe de distinguer deux types différents de transferts de technologie dans le domaine de la biotechnologie: le premier consiste en un transfert direct (importation, achat direct) d'un produit fini issu de la biotechnologie (une nouvelle variété transgénique par exemple) et le second implique l'utilisation de la biotechnologie à titre d'instrument dans la recherche agricole (c'est le cas des marqueurs génétiques utilisés dans la sélection végétale). Les droits de propriété intellectuelle peuvent avoir sur le transfert de technologie, qu'il s'agisse du transfert direct de produits ou de l'utilisation de biotechnologies dans la recherche et le développement, des conséquences dont la forme et l'importance peuvent varier grandement. 


\subsection{CONSÉQUENCES POUR LES AGRICULTEURS ET POUR LES AUTRES CONSOMMATEURS FINAUX}

Le principal argument avancé en faveur d'un renforcement des droits de propriété intellectuelle est qu'il stimulera l'investissement et l'innovation. Si cet argument se vérifie, on peut prévoir que le transfert de technologie mettra à la disposition des agriculteurs et d'autres consommateurs finaux un choix plus vaste de technologies qui devrait en principe avoir des conséquences positives pour les producteurs agricoles. Dans le même temps, des sociétés hésiteront peut-être à transférer des technologies exclusives vers des pays qui ne possèdent pas un système efficace de protection des droits de propriété intellectuelle.

L'expérience réunie jusqu'ici étant très récente et limitée à un petit nombre de pays (comprenant l'Australie, le Canada, les Etats-Unis et la France), il est difficile d'identifier des tendances précises (Brenner, 1997) dans l'évolution des prix des nouvelles variétés transgéniques. Dans certains cas, le prix des semences transgéniques ne dépasse pas celui des semences traditionnelles; dans d'autres, il comprend une «prime technologique». On peut toutefois affirmer que dans l'ensemble le coût des nouvelles variétés transgéniques sera supérieur à celui des variétés obtenues par des méthodes traditionnelles. L'agriculteur qui achète les nouvelles variétés transgéniques devra alors mettre en balance, d'une part, le prix plus élevé des semences et, d'autre part, les avantages de cette nouvelle variété (qualité, rendement, résistance aux ravageurs ou aux maladies). Il importe peu à l'agriculteur de savoir si les semences sont protégées par des droits de propriété intellectuelle, sauf si cette protection limite ses droits antérieurs, c'est-à-dire le droit de conserver, de réutiliser ou d'échanger des semences récoltées.

A cet égard, l'expérience faite en l'Inde avec les semences de maïs hybride rappelons que l'obtenteur d'hybrides jouit de droits semblables à ceux d'un titulaire de brevet - est on ne peut plus explicite. Bien que les prix des semences hybrides produites par le secteur privé soient considérablement plus élevés que ceux de variétés améliorées pollinisées à l'air libre produites par le secteur public, le volume des ventes de semences hybrides du secteur privé a suivi une croissance beaucoup plus rapide que le volume des ventes des variétés améliorées. Actuellement, le secteur privé occupe $50 \%$ du marché des semences de maïs, ce qui donne à penser que les entreprises privées assurent des livraisons plus efficaces aux agriculteurs et/ou qu'elles leur fournissent des semences de qualité plus constante (Singh et al., 1995).

\subsection{CONSÉQUENCES POUR L'INNOVATION LOCALE}

Pour les pays en développement, l'un des grands enjeux dans ce domaine est de savoir si la protection de la propriété intellectuelle conférera plus de poids aux innovateurs locaux et, par conséquent, si elle renforcera la capacité du pays à innover.

Tant dans les pays de l'OCDE que dans les pays en développement, le rôle des secteurs public et privé dans l'innovation agricole, de même que l'équilibre entre ces deux secteurs, sont en train d'évoluer. Dans les pays de l'OCDE, le financement de la recherche et de l'innovation agricoles par le secteur public a 
en général diminué, voire stagné, ces dernières années. Dans le même temps, les investissements du secteur privé ont augmenté. Pendant la période allant de 1981 à 1993, la recherche privée dans le domaine agricole a, par exemple, enregistré une croissance annuelle moyenne de 5,1\% et représentait à la fin de cette période environ la moitié des dépenses totales consacrées à la recherche agricole (Pardey, 1997). L'investissement privé a surtout revêtu une importance particulière dans le domaine des biotechnologies.

Les données dont nous disposons indiquent que les droits de propriété intellectuelle stimulent les investissements du secteur privé dans la recherche agricole (Thirtle et Echeverria, 1994; Fuglie et al., 1996). Elles montrent toutefois aussi que les investissements privés dans la sélection végétale vont se limiter à un petit groupe de cultures commerciales telles que le coton, le maïs, le soja, les légumes et le blé. En effet, à moins de faire l'objet de mesures d'incitation spécifiques, les sociétés privées ne vont sans doute pas investir dans la recherche et le développement de variétés pollinisées à l'air libre ni de végétaux cultivés par des agriculteurs manquant de ressources.

\subsection{CONSÉQUENCES POUR LA RECHERCHE PUBLIOUE}

Pour la recherche publique dans l'agriculture, l'introduction des droits de propriété intellectuelle aura sans doute des retombées aussi bien positives que négatives. D'une part, il faut savoir que dans nombre de pays en développement, le secteur public continue de jouer un rôle central non seulement dans la recherche agricole en général - et dans la recherche biotechnologique en particulier - mais souvent aussi pour ce qui est du développement de technologies et de leur diffusion. Cependant, les investissements publics destinés à la recherche agricole ont stagné, voire décliné, sous l'effet des politiques d'ajustement structurel et de libéralisation appliquées par un nombre croissant de pays. Par conséquent, on tend à exiger des instituts de recherche publics qu'ils s'impliquent dans des activités génératrices de revenu. L'introduction des droits de propriété intellectuelle pourrait donc dans une certaine mesure avoir un effet positif, puisqu'elle engendrerait une hausse des revenus provenant de la concession de licences, de redevances ou de la prestation de services. Cette hausse serait d'autant plus marquée pour les instituts publics qui jusqu'ici ont mis gratuitement leurs «innovations» à la disposition tant du secteur public que du secteur privé (van Wikj et Jaffé, 1995).

Il convient d'autre part de souligner que la recherche agricole et le processus d'innovation des pays en développement ont jusqu'ici tiré grand profit de la formation offerte par les universités et les instituts de recherche des pays industrialisés et de la collaboration menée avec eux dans le domaine expérimental. Les milieux scientifiques des pays de l'OCDE s'inquiètent à présent de voir s'éroder le libre échange d'informations et de résultats scientifiques qui caractérisait la recherche académique il y a peu encore. Cette érosion s'explique pour deux raisons: premièrement, les instituts de recherche publics dépendent de plus en plus de financements privés et, deuxièmement, une partie toujours plus grande des résultats scientifiques sont protégés, puisque l'université elle-même ou son (ses) partenaire (s) privé (s) exigent qu'ils bénéficient des droits de propriété intellectuelle. Cette évolution engendre des conséquences évidentes pour les scientifiques des pays en développement. 
Dans le débat sur l'introduction des droits de propriété intellectuelle, certains ont exprimé leur crainte de voir l'extension de ces droits au matériel génétique entraver les échanges de germoplasmes, qui sont un élément essentiel dans la recherche et le développement dans l'agriculture. A cela, les défenseurs des droits de propriété intellectuelle rétorquent que la protection accroîtra le transfert de matériel génétique des pays industrialisés vers les pays en développement, mais cela reste à prouver. Etant donné que le secteur privé est très présent dans les biotechnologies, on peut prévoir que les nouvelles biotechnologies seront de plus en plus protégées par une forme ou l'autre de droits de propriété intellectuelle. Dans ce cas, l'introduction de ces droits augmentera certes la part de matériel génétique transférée dans un contexte commercial, mais qu'en serat-il des échanges non commerciaux (ou de biens publics)?

Les voies permettant d'accéder aux technologies brevetées sont très variées et font intervenir aussi bien des sources commerciales que non commerciales. Outre l'achat direct ou l'acquisition de licences, la consultation des registres des brevets - en raison de l'obligation de divulgation - peut constituer une source précieuse d'information technologique. De plus, tant la législation sur les brevets que les droits de propriété intellectuelle prévoient une exception en faveur de la recherche, de sorte que les matériels sur lesquels se fonde une invention protégée sont mis à disposition aux fins de recherche.

Voici divers moyens qui permettent de faciliter l'accès aux technologies protégées: programmes et projets publics menés conjointement, par exemple au niveau des universités; coopération au développement bilatérale ou multilatérale dans le cadre des centres internationaux de recherche agricole (réseau du Groupe consultatif de la recherche agricole internationale, GCRAI). Il est aussi possible de faire intervenir des organismes tels que l'International Service for the Acquisition of Agri-Biotech Applications (ISAAA) qui servent d'intermédiaires ou de "courtiers » dans le transfert ou la donation de technologies protégées au secteur public.

Voici deux exemples de ce genre de donations: l'accord passé entre l'ISAAA, Monsanto et le Centre of Research and Advanced Studies (CINVESTAV) au Mexique; l'accord entre Ciba-Geigy (désormais Novartis) et l'Institut international de recherches sur le riz (IRRI) aux Philippines. (L'institut IRRI est l'un des centres internationaux de recherche agricole disséminés de par le monde.) Le premier de ces accords réglait le transfert d'une technique génétique développée par Monsanto (qui rend les pommes de terre résistantes aux virus) à un institut de recherche public au Mexique. Le second régit le transfert d'un gène synthétique Bacillus thuringiensis (Bt.) de résistance aux insectes, breveté par Ciba-Geigy, à l'IRRI en vue d'une utilisation dans du riz transgénique. Dans les deux cas, le transfert a pris la forme d'une licence gratuite. Dans le deuxième cas, le riz obtenu portant le gène $B t$. ne pourra pas être vendu aux producteurs d'Australie, du Canada, des Etats-Unis, du Japon, de Nouvelle-Zélande et des pays signataires de la Convention sur le brevet européen.

Jusqu'ici, on n'a assisté qu'à de rares donations de technologies de ce genre, avec ou sans l'intervention d'un intermédiaire, et les exemples connus sont encore trop récents pour que l'on puisse évaluer le succès de ces initiatives.

En bref, les échanges de germoplasmes ne seront bientôt plus régis par le principe de la libre circulation, mais interviendront sur la base de différents types 
d'accords juridiques et/ou commerciaux. Reste à connaître l'impact de ces nouveaux accords de transfert de technologie sur le volume des échanges.

Dans l'ensemble, que l'on se place du point de vue des agriculteurs, des inventeurs ou de la recherche publique, l'introduction ou le renforcement des droits de propriété intellectuelle dans le domaine de la biotechnologie et de l'agriculture auront des effets aussi bien positifs que négatifs. Dans ce contexte, on doit se demander quelle place occuperont les centres internationaux de recherche agricole du GCRAI, plus connus sous le nom de réseau du Groupe consultatif. Ces centres ont en effet joué un rôle central dans le transfert des technologies de la «Révolution verte»- variétés à haut rendement, en particulier de blé et de riz - vers les pays en développement.

Ceux de ces centres qui ont conclu un accord avec la FAO en octobre 1994 sont les gardiens des ressources phytogénétiques qui figurent dans leurs collections et qu'ils conservent au profit de la communauté internationale. Par le passé, ces centres mettaient aussi bien les germoplasmes conservés dans les banques de gènes que les matériels issus de leurs propres recherches librement à la disposition des pays développés et en développement, ainsi que des instituts de recherche et des sociétés tant publics que privés. L'extension des droits de propriété intellectuelle soulève des problèmes délicats pour le réseau du Groupe consultatif, puisqu'elle permet à des tiers d'obtenir soit un brevet, soit des droits d'obtention sur des variétés obtenues à partir du matériel fourni par les centres. Dans le même temps, les centres risquent d'être confrontés à un dilemme lorsqu'ils auront besoin de se procurer du matériel perfectionné ou des techniques de recherche de pointe susceptibles de faire avancer leurs efforts de recherche de manière importante, mais qui auront été brevetés par des sociétés privées.

Le renforcement des droits de propriété intellectuelle soulève la question suivante: dans quelle mesure un bon système public de recherche et de transfert de technologie en faveur des pays en développement, tel qu'il a prévalu dans le cas de la Révolution verte, pourra-t-il coexister avec un système mondial basé sur la protection des technologies?

\section{CONCLUSIONS}

Dans les chapitres ci-dessus, nous avons pris le cas des biotechnologies dans le domaine de l'agriculture pour analyser les conséquences que pourra avoir le renforcement des droits de propriété intellectuelle pour les pays en développement. Il importe toutefois de souligner que l'impact des droits de propriété intellectuelle sur le transfert de technologies et d'inventions dans les pays en développement n'a guère été étudié. Relevons également que l'introduction de ces droits ne suffira pas à elle seule pour promouvoir le transfert de technologies et d'innovations sans la mise en œuvre de toute une série de mesures et d'institutions connexes.

En fait, certains chercheurs vont même jusqu'à douter des avantages que l'introduction des droits de propriété intellectuelle pourra apporter aux pays en développement. Un auteur a tenté d'évaluer l'impact sur le bien-être des pays d'une généralisation des droits de propriété intellectuelle au niveau mondial. Pour ce faire, il a utilisé le cas d'un simple modèle breveté pour analyser les répercussions d'une 
extension de la protection du brevet du pays «inventeur» au pays «consommateur». Voici sa conclusion: plus le nombre des pays auxquels s'étend la protection des brevets est grand, plus les effets globaux de cette extension diminuent au point de devenir négatifs. Il serait par conséquent judicieux d'exempter certains pays, au moins les plus pauvres, de l'obligation d'adhérer à un nouvel accord d'extension de la protection des brevets (Deardoff, 1992).

Une étude exhaustive de la littérature spécialisée (Siebeck et al., 1990) conclut que les droits de propriété intellectuelle engendreront aussi bien des coûts que des revenus pour les pays en développement et que ces coûts et ces revenus varieront d'un pays à l'autre et, très probablement aussi, d'un secteur à l'autre. Voici les principaux facteurs qui les détermineront: la réaction des inventeurs nationaux face à une meilleure protection, la réaction des investisseurs directs étrangers et la demande de produits protégés.

Malgré la tendance à un renforcement et une extension des droits de propriété intellectuelle, une grande part des technologies connues appartiennent au domaine public, soit parce que l'on n'a jamais cherché à les breveter, soit parce que la durée de protection est échue. Il faut toutefois se rendre à l'évidence: après la conclusion de l'ADPIC, de plus en plus de technologies bénéficieront d'une protection et une part croissante des transferts de technologies se dérouleront dans un contexte commercial. Que deviendront donc les technologies de «bien public» dont les pays en développement ont cruellement besoin - dans les domaines de la santé publique, de la protection de l'environnement, de l'alimentation et de l'agriculture - mais qui ne suscitent qu'une demande (au sens économique) limitée, tout au moins à court terme?

Il importe surtout que les organismes engagés dans des projets et des programmes qui visent à développer ou à transférer des technologies relevant du «bien public» vers les pays en développement - organismes de coopération bilatéraux ou multilatéraux, fondations privées, ONG - soient conscients des difficultés que risque de poser une large application des droits de propriété intellectuelle. Des organismes avisés seront en effet mieux à même de prévoir les obstacles et les contraintes possibles et d'élaborer des stratégies pour les combattre ou les surmonter.

De leur côté, les gouvernements des pays en développement ont aussi un rôle important à jouer: mettre en place un cadre juridique adéquat pour régir les droits de propriété intellectuelle et formuler des politiques appropriées pour stimuler le transfert de technologie et d'innovations. Bien que les choix politiques soient loin d'être simples et aisés, voici les éléments clés du rôle de ces gouvernements.

\subsection{RESPECTER LES ENGAGEMENTS DE L'ADPIC}

La révision de l'article 27 (3b) de l'accord ADPIC, qui régit la protection des variétés végétales, est prévue en janvier 1999. Les pays en développement disposent donc de très peu de temps pour faire leur choix et pour mettre sur pied des systèmes de protection qui répondent à leurs besoins spécifiques tout en respectant leurs intérêts. Une première tentative visant à définir et à évaluer les éléments qui pourraient constituer un système sui generis envisageable qui soit compatible avec l'accord ADPIC et puisse remplacer l'adhésion à la Conven- 
tion de l'UPOV a été menée sous les auspices de l'International Plant Genetic Resources Institute, IPGRI (Leskien et Flitner, 1997).

Pour appliquer les nouveaux droits de propriété intellectuelle, certains pays pourront se contenter de modifier légèrement les lois existantes, mais d'autres devront peut-être aller jusqu'à créer de nouvelles institutions et de nouvelles structures. Après avoir mis en place la législation adéquate, les pays devront aussi veiller à son application et à son respect. Ce travail exige des ressources financières ainsi qu'un savoir-faire technique et juridique.

\subsection{CRÉER UN CADRE POLITIQUE APPROPRIÉ POUR LES INNOVATIONS LOCALES}

Une protection renforcée de la propriété intellectuelle devrait, entre autres, stimuler le secteur privé de la recherche et de l'innovation. Il n'en reste pas moins que la législation sur les droits de propriété intellectuelle devra être appliquée et que cette application relève en général d'institutions nationales (offices nationaux des brevets ou, dans le cas des droits d'obtention, d'institutions du secteur des semences). Or, dans nombre de pays ces institutions manquent encore de moyens pour s'acquitter de cette tâche. Outre la législation sur les droits de propriété intellectuelle, il s'agira de créer un cadre réglementaire explicite et efficace pour assurer le transfert sans danger des produits des nouvelles biotechnologies et rares sont les pays en développement qui possèdent aujourd'hui des directives ou une législation sur la biosécurité.

Les pays dont le marché des technologies est sous-développé devront prendre des mesures complémentaires pour inciter le secteur privé à s'engager d'avantage. Il s'agira aussi de multiplier les efforts pour stimuler le secteur privé en mettant sur pied des partenariats entre les secteurs public et privé.

\subsection{DÉFINIR UN RÔLE APPROPRIÉ POUR LE SECTEUR PUBLIC}

Si l'on veut encourager l'innovation nationale, on devra à l'avenir mieux cibler la recherche publique. Puisque l'on ne peut guère s'attendre à court terme à une augmentation du total des investissements publics consacrés à la recherche (tant pour ce qui est des financements de l'Etat que des donateurs), il est d'autant plus important d'utiliser avec le maximum d'efficacité les rares ressources publiques disponibles. Dans certains pays, le secteur privé joue déjà un rôle plus actif dans la recherche et le développement et il convient de soutenir cette tendance.

Cependant, même après l'introduction des droits de propriété intellectuelle, certains domaines de recherche et secteurs économiques ne seront pas en mesure, à court terme, d'attirer des investissements privés. Le secteur public devrait donc opérer sur deux plans en matière de recherche et d'innovation: d'une part, collaborer dans la mesure du possible avec le secteur privé en l'incitant à s'engager d'avantage et, d'autre part, concentrer les efforts de recherche sur des domaines importants du point de vue social et environnemental, mais qui ne sont pas en mesure de générer des bénéfices à court terme et offrent donc peu d'attrait pour les investisseurs privés. 


\subsection{NÉGOCIER DES ACCORDS DE TRANSFERT DE TECHNOLOGIE}

Même lorsqu'une technologie est protégée, les gouvernements et les organes publics disposent d'une certaine marge de manœuvre pour négocier un transfert. En effet, aucune disposition de l'ADPIC n'empêche un gouvernement (ou un donateur ou une institution financière internationale) de négocier des conditions avantageuses et/ou d'accorder une aide financière et technique pour permettre le transfert d'une technologie protégée à des conditions préférentielles.

Les gouvernements ont aussi la possibilité de faire appel aux services d'un « courtier honnête» (c'est-à-dire un organisme tel que l'ISAAA, une ONG compétente ou un expert indépendant) pour servir d'intermédiaire dans la négociation des conditions d'un transfert de technologie entre un gouvernement, les autorités et des sociétés privées.

\section{BIBLIOGRAPHIE}

Brenner, Carliene (1996), Introduire la biotechnologie dans l'agriculture: Incitations, obstacles, expériences, Etudes du Centre de développement, OCDE, Paris.

Brenner, Carliene (1997), Biotechnology Policy for Developing Country Agriculture, Policy Brief, $\mathrm{n}^{\circ} 14$, Centre de développment, OCDE, Paris.

Brenner, Carliene, Intellectual Property Rights and Technology Transfer in Developing Country Agriculture: Rhetoric and Reality, Centre de développement, OCDE, Paris, à paraître.

Deardorff, Alan V. (1992), «Welfare Effects of Global Patent Potection », in Economica, vol. 59, février.

Echeverria, Ruben, Eduardo J. Trigo et Derek Byerlee (1996), Institutional Change and Effective Financing of Agricultural Research in Latin America, Banque mondiale, Technical Paper, ${ }^{\circ}$ 330, Washington, D.C. août.

Fuglie, Keith, Nicola Ballenger, Kelly Day, Cassandra Klotz, Michael Ollinger, John Reilly, Uptal Vasavada et Jet Yee (1996), Agricultural Research and Development: Public and Private Investments under Alternative Markets and Institutions, Etats-Unis, Département de l'agriculture, AER, $\mathrm{n}^{\circ} 735$, Washington, mai.

Joly, Pierre-Benoît et Marie-Angèle de Looze (1996), «An Analysis of Innovation Strategies and Industrial Differentiation through Patent Applications : the Case of Plant Biotechnology », in Research Policy, vol. 25.

Juma, Calestous (1989), The Gene Hunters: Biotechnology and the Scramble for Seed, Princeton University Press, Princeton.

Leskien, Dan et Michael Flitner (1997), Intellectual Property Rights and Plant Genetic Resources: Options for a Sui Generis System, IFPRI, Issues in Genetic Resources, ${ }^{\circ} 7$, Rome.

Lesser, W. (1991), Equitable Patent Protection in the Developing World: Issues and Approaches, Eubios Ethics Institute, Nouvelle-Zélande.

Lesser, W. (1994), Institutional Mechanisms supporting Trade in Genetic Materials, PNUE, Environment and Trade Series, $\mathbf{n}^{\circ} 4$, Genève.

Lesser, W. (1997), Assessing the Implications of IPR on Plant and Animal Agriculture, Staff Paper 97-04, Cornell University, Ithica, Etats-Unis, juin.

OCDE (1996), Propriété intellectuelle, transfert de technologie et ressources génétiques, Paris.

Pardey, Phillip (1997), IFRI, Principles, Policies and Institutional Aspects of Financing Agricultural R \& D, paper presented at the workshop on «New Investment Strategies for Agricultural dans Natural Resources Research", Londres, 1 - 2 octobre 1997.

Siebeck, Wolfgang E. avec Robert Evenson, William Lesser et Carlos A. Primo Braga (1990), Strengthening Protection of Intellectual Property in Developing Countries: a Survey of the Literature, Banque mondiale, Discussion Paper, $\mathrm{n}^{\circ} 112$.

Singh, R.P., Suresh Pal et Michael Morris (1995), Maize Research, Development and Seed Production in India: Contributions of the Public and Private Sector, Economics Working Paper 9503, CIMMYT, Mexico.

Thirtle, Colin et Ruben G. Echeverria (1994), Privatisation and the Roles of Public and Private Institutions in Agricultural Research in sub-Saharan Africa, in: Food Policy, vol. 19, $\mathrm{n}^{\circ} 1$, février.

van Wijk, Jeroen et Walter Jaffé (éd.), Proceedings of a Seminar on the Impact of Plant Breeders'Rights in Developing Countries, March 7-8, 1995, Bogotá, Colombie. 